\title{
Frequent wheeze at follow up of very preterm infants: which factors are predictive?
}

\section{Thomas, A Greenough, A Johnson, E Limb, N Marlow, J L Peacock, S Calvert}

See end of article for authors' affiliations

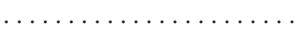

Correspondence to: Professor Greenough Department of Child Health, King's College Hospital, London SE5 9RS, UK;

anne.greenough@kcl.ac.uk

Accepted 14 September 2002

\begin{abstract}
Objective: To determine if chest radiograph appearance at 28 days or 36 weeks postmenstrual age (PMA) can predict recurrent wheeze or cough at follow up in prematurely born infants more effectively than readily available clinical data.

Design: Chest radiographs of infants entered into the UKOS trial, who had had a chest radiograph at 28 days and 36 weeks PMA and completed six months of follow up, were assessed for the presence of fibrosis, interstitial shadows, cystic elements, and hyperinflation. At 6 months of corrected age, the occurrence and frequency of wheeze and cough since discharge were determined using a symptom questionnaire.

Patients: A total of 185 infants with a median gestational age of 26 (range 23-28) weeks.

Results: Thirty seven infants wheezed more than once a week, compared with the rest of the cohort. These infants had significantly higher chest radiograph scores at 28 days $(p=0.020)$ and 36 weeks PMA ( $p=0.005)$, with significantly higher scores at 28 days for fibrosis $(p=0.017)$ and at 36 weeks PMA for fibrosis $(p=0.001)$ and cystic elements $(p=0.0007)$. They had also been ventilated for longer $(p=0.013)$. Forty four infants coughed more than once a week; they did not differ significantly from the rest of the cohort. An abnormal chest radiograph score at 36 weeks PMA had the largest area under the receiver operator characteristic curve with regard to prediction of frequent wheeze.

Conclusion: An abnormal chest radiograph appearance at 36 weeks PMA predicts frequent wheeze at follow up and appears to be a better predictor than readily available clinical data.
\end{abstract}

$\mathrm{P}$ reterm infants often have troublesome wheezes and coughs at follow up. ${ }^{1}$ Affected infants have appreciable lung function abnormalities, increased airways resistance, and low functional residual capacity to thoracic gas volume ratio, indicating gas trapping. ${ }^{2}$ Bronchodilator treatment relieves the symptoms, ${ }^{3}$ but many infants also require prophylactic agents such as sodium cromoglycate ${ }^{4}$ and inhaled steroids. ${ }^{5}$ Early identification of infants at high risk of chronic respiratory morbidity would facilitate targeted interventions.

Infants born at very early gestations and/or who had prolonged dependency on ventilatory support and supplementary oxygen are most likely to have troublesome symptoms at follow up. ${ }^{1}$ We hypothesised that chest radiograph appearance at 28 days or 36 weeks postmenstrual age (PMA) would predict such an adverse outcome, as the 28 day chest radiograph appearance has been reported to have $88 \%$ sensitivity and $71 \%$ specificity in predicting infants with the most severe lung function abnormalities at 6 months of age. ${ }^{6}$ In addition, chest radiograph appearance at 36 weeks PMA differed significantly between those who did and did not remain dependent on supplementary oxygen. ${ }^{7}$ The aim of this study was to determine if chest radiograph appearance at 28 days or 36 weeks PMA predicted frequent wheeze or cough at follow up in very prematurely born infants and was a better predictor than readily available clinical data. A subsidiary aim was to determine if chest radiograph appearance was a useful predictor of wheeze or cough in non-oxygen dependent infants, as this would be useful information when considering whether such infants should have a routine chest radiograph at 36 weeks PMA.

\section{METHODS}

Infants in the United Kingdom oscillation study (UKOS) trial were included in this study if they had had chest radiographs taken routinely at 28 days and 36 weeks PMA and had completed six months of follow up as per the UKOS protocol. The UKOS trial was a randomised study comparing high frequency oscillation (HFO) with conventional ventilation. Anteroposterior chest radiographs taken in the supine position were assessed using a previously described scoring system, ${ }^{8}$ by a researcher (AG) who was unaware of the infants' outcomes. The radiographs were assessed for the presence of fibrosis/interstitial shadows, cystic elements, and the degree of hyperinflation. Fibrosis/interstitial shadows were defined as focal areas of linear or nodular density within the lungs without a normal anatomical origin. The presence of such shadowing was scored on a zonal basis, the chest being divided into four zones (right and left, upper and lower). A score of one was given for each zone in which there was fibrosis/interstitial shadows. Cysts were defined as lucent intraparenchymal lesions with a well circumscribed margin and scored as to whether they were absent (score 0), small (less than one third of the ipsilateral lung field; score 1) or multiple and large (score 2). The degree of expansion of the lungs was determined by counting the posterior ends of the ribs on both sides of the chest visible above the diaphragm (less than 14 ribs, score $=0$; $14-16$ ribs, score $=1$; more than 16 ribs, score $=2$ ). The maximum overall score was 8 . The reproducibility of the scoring system had been previously determined, and the mean (SD) difference between paired scores was $0.1(0.64){ }^{8}$

As part of the UKOS trial, the infants' gestational age, birth weight, duration of ventilation, and oxygen dependency were recorded. At 6 months of age corrected for prematurity, all the infants were seen by their local paediatrician. A standard

Abbreviations: PMA, postmenstrual age; UKOS, United Kingdom oscillation study; HFO, high frequency oscillation; ROC, receiver operator characteristic 
Table 1 Comparison of infants who did and did not frequently wheeze

\begin{tabular}{|c|c|c|c|}
\hline & $\begin{array}{l}\text { Frequent wheeze } \\
(n=37)\end{array}$ & $\begin{array}{l}\text { Non-frequent wheeze } \\
(n=148)\end{array}$ & $\mathrm{p}$ Value \\
\hline Gestational age (weeks) & $26.6,26.4(23.6-28.9)$ & $26.6,26.8(23.0-28.9)$ & 0.95 \\
\hline Birth weight $(g)$ & $899,870(590-1459)$ & $882,873(500-1430)$ & 0.81 \\
\hline \multicolumn{4}{|l|}{ Maternal smoking (\%) } \\
\hline In pregnancy & 22 & 20 & 0.83 \\
\hline After birth & 20 & 22 & 0.84 \\
\hline Family history of atopy (\%) & 49 & 57 & 0.34 \\
\hline \multicolumn{4}{|l|}{ Duration (days) of } \\
\hline Ventilation & $25.6,21(1-75)$ & $17.3,11.5(0-112)$ & 0.013 \\
\hline Supplementary oxygen & $84.4,67$ (20-239) & $69.3,73(1-179)$ & 0.42 \\
\hline \multicolumn{4}{|l|}{ Oxygen dependency (\%) } \\
\hline At 28 days & 97 & 87 & 0.08 \\
\hline At 36 weeks & 76 & 70 & 0.47 \\
\hline At discharge & 43 & 29 & 0.09 \\
\hline \multicolumn{4}{|c|}{ Chest radiograph score at 28 days } \\
\hline Total & $4.2,4(1-8)$ & $3.4,3(0-7)$ & 0.020 \\
\hline Fibrosis & $2.9,3.0(1-4)$ & $2.4,2(0-4)$ & 0.017 \\
\hline Cystic elements & $0.2,0(0-2)$ & $0.1,0(0-2)$ & 0.17 \\
\hline Hyperinflation & $1.1,1(0-2)$ & $0.9,1(0-2)$ & 0.17 \\
\hline \multicolumn{4}{|c|}{ Chest radiograph score at 36 weeks PMA } \\
\hline Total & $4.5,5(0-8)$ & $3.5,3(0-7)$ & 0.005 \\
\hline Fibrosis & $3.1,4(0-4)$ & $2.4,2(0-4)$ & 0.001 \\
\hline Cystic elements & $0.2,0(0-2)$ & $0.1,0(0-1)$ & 0.0007 \\
\hline Hyperinflation & $1.1,1(0-2)$ & $1.1,1(0-2)$ & 0.67 \\
\hline
\end{tabular}

questionnaire was administered by the paediatrician. The parents were asked if their infant coughed and/or wheezed more than once a week, more than once a month but less than once a week, less than once a month, or not at all. In addition, information was collected on whether there was a family history of atopy (parents or siblings had asthma, eczema, or hay fever) and whether the mother had smoked during and/or after pregnancy.

\section{Analysis}

Frequent wheeze or cough was defined as wheeze or cough occurring more than once a week. Differences between infants with frequent wheeze or cough and the rest of the cohort were assessed for statistical significance using the Wilcoxon rank sum or $\chi^{2}$ tests as appropriate. Receiver operator characteristic (ROC) plots were constructed, ${ }^{9}$ and the areas below the ROC curves compared ${ }^{10}$ to determine which predictive factor performed best. Subgroup analysis was performed to determine the best predictor of frequent wheeze or cough in the group of infants who were non-oxygen dependent at 36 weeks PMA.

\section{Patients}

In the UKOS trial, 440 of 797 infants had had a chest radiograph at 28 days, and 333 at 36 weeks PMA. The 185 infants, with median gestational age $26^{+5}$ weeks (range $23^{+0}-28^{+6}$ ) and median birth weight $870 \mathrm{~g}$ (range 500-1459), who had had chest radiographs at 28 days and 36 weeks PMA and follow up within the predefined window - that is, between five and eight months of age corrected for prematurity-were included in this study. A total of 131 of the 185 infants $(71 \%)$ were oxygen dependent at 36 weeks PMA, compared with $55 \%$ of survivors in the UKOS trial overall. The UKOS trial was approved by the multicentre research ethics committee and the local research ethics committees. All parents gave informed written consent for their infants to take part in the trial.

\section{RESULTS}

Infants with frequent wheeze $(\mathrm{n}=37)$ differed significantly from the rest of the cohort in having a higher total chest radiograph score at 28 days $(\mathrm{p}=0.020)$ and 36 weeks PMA $(\mathrm{p}=0.005)$, with higher scores at 28 days for fibrosis $(\mathrm{p}=0.017)$ and at 36 weeks PMA for fibrosis/interstitial shadows $(p=0.001)$ and cystic elements $(p=0.0007)$. Infants with frequent wheeze also required ventilation for longer $(p=0.0013)$ (table 1$)$. As the duration of ventilation and the total chest radiograph score were both significantly associated with frequent wheeze, logistic regression was performed to investigate the relation between them. When both the total chest radiograph score and the duration of ventilation were analysed together, the relation with the duration of ventilation was weaker and became non-significant (adjusted odds ratio 1.01 (95\% confidence interval 0.99 to $1.03), \mathrm{p}=0.172$ ). In this two variable model, however, the relation with the total chest radiograph score remained significant (adjusted odds ratio 1.31 (95\% confidence interval 1.03 to 1.69$), \mathrm{p}=0.027$ ).

There were no significant differences between infants who did $(n=44)$ and did not cough frequently (table 2 ).

Comparison of areas under the ROC curves suggested that the total chest radiograph score at 36 weeks PMA was the best predictor of frequent wheeze (table 3). Comparison of ROC curves confirmed this, showing that the area under the curve for the 36 week PMA chest radiograph score was significantly larger than that for birth weight $(p=0.01)$, maternal smoking in pregnancy $(p=0.03)$ or after birth $(p=0.05)$, family history of atopy $(p=0.01)$, and oxygen dependency at 36 weeks PMA $(p=0.02)$, but not significantly greater than that for gestational age $(p=0.18)$, duration of ventilation $(p=0.85)$ or oxygen dependency at 28 days $(p=0.06)$, or oxygen dependency at discharge $(p=0.19)$. In contrast, there were no significant predictors of frequent cough (table 4). In the infants who were not oxygen dependent at 36 weeks PMA, the presence of fibrosis on the chest radiograph taken at 36 weeks had the largest area under the ROC curve, with regard to frequent wheeze, but the numbers were too small for further analysis in this subset (table 5). There were no significant predictors of frequent cough in that group (data not shown). A total chest radiograph score of three or more had $81 \%$ sensitivity and $28 \%$ specificity in predicting frequent wheeze.

\section{DISCUSSION}

We have shown that very prematurely born infants who frequently wheeze, but not cough, at follow up have significantly worse chest radiograph abnormalities than those 
Table 2 Comparison of infants who did and did not frequently cough

\begin{tabular}{|c|c|c|c|}
\hline & $\begin{array}{l}\text { Frequent cough } \\
(n=44)\end{array}$ & $\begin{array}{l}\text { Non-frequent cough } \\
(n=141)\end{array}$ & $\mathrm{p}$ Value \\
\hline Gestational age (weeks) & $26.5,26.6(23.6-28.9)$ & $26.6,26.9(23.0-28.9)$ & 0.47 \\
\hline Birth weight (g) & $898,889(580-1360)$ & 881,865 (500-1459) & 0.81 \\
\hline \multicolumn{4}{|l|}{ Maternal smoking (\%) } \\
\hline In pregnancy & 23 & 19 & 0.59 \\
\hline After birth & 20 & 22 & 0.76 \\
\hline \multicolumn{4}{|l|}{ Duration (days) of } \\
\hline Ventilation & $22.0,16.5(0-75)$ & $18.0,12(0-112)$ & 0.22 \\
\hline Oxygen & $81.2,78(2-239)$ & $69.5,70(1-179)$ & 0.41 \\
\hline Family history of atopy (\%) & 45 & 59 & 0.12 \\
\hline \multicolumn{4}{|l|}{ Oxygen dependency (\%) } \\
\hline At 28 days & 91 & 89 & 0.67 \\
\hline At 36 weeks & 77 & 69 & 0.28 \\
\hline At discharge & 39 & 30 & 0.26 \\
\hline \multicolumn{4}{|l|}{$\begin{array}{l}\text { Chest radiograph score at } 28 \\
\text { days }\end{array}$} \\
\hline Total & $3.8,4(0-8)$ & $3.4,3(0-7)$ & 0.31 \\
\hline Fibrosis & $2.7,2.5(0-4)$ & $2.4,2(0-4)$ & 0.19 \\
\hline Cystic elements & $0.2,0(0-2)$ & $0.1,0(0-2)$ & 0.44 \\
\hline Hypertension & $0.9,1(0-2)$ & $0.9,1(0-2)$ & 0.94 \\
\hline \multicolumn{4}{|c|}{ Chest radiograph score at 36 weeks PMA } \\
\hline Total & $4.0,4(0-8)$ & $3.6,3(0-7)$ & 0.15 \\
\hline Fibrosis & $2.8,3(0-4)$ & $2.5,2(0-4)$ & 0.12 \\
\hline Cystic elements & $0.2,0(0-2)$ & $0.1,0(0-1)$ & 0.12 \\
\hline Hyperinflation & $1.1,1(0-2)$ & $1.0,1(0-2)$ & 0.77 \\
\hline
\end{tabular}

\begin{tabular}{|c|c|c|}
\hline Predictor & ROC area & $\mathrm{p}$ Value \\
\hline Gestational age & 0.50 & 0.95 \\
\hline Birth weight & 0.49 & 0.81 \\
\hline \multicolumn{3}{|l|}{ Maternal smoking } \\
\hline In pregnancy & 0.51 & 0.83 \\
\hline After birth & 0.49 & 0.84 \\
\hline Family history of atopy & 0.46 & 0.34 \\
\hline \multicolumn{3}{|l|}{ Duration of } \\
\hline Ventilation & 0.63 & 0.01 \\
\hline Supplementary oxygen & 0.56 & 0.42 \\
\hline \multicolumn{3}{|l|}{ Oxygen dependency } \\
\hline At 28 days & 0.55 & 0.08 \\
\hline At 36 weeks & 0.53 & 0.47 \\
\hline At discharge & 0.58 & 0.08 \\
\hline \multicolumn{3}{|c|}{ Chest radiograph score at 28 days } \\
\hline Total & 0.62 & 0.02 \\
\hline Fibrosis & 0.62 & 0.02 \\
\hline Cystic elements & 0.54 & 0.17 \\
\hline Hyperinflation & 0.57 & 0.17 \\
\hline \multicolumn{3}{|c|}{ Chest radiograph score at 36 weeks PMA } \\
\hline Total & 0.65 & 0.005 \\
\hline Fibrosis & 0.67 & 0.001 \\
\hline Cystic elements & 0.59 & 0.007 \\
\hline Hyperinflation & 0.52 & 0.67 \\
\hline
\end{tabular}

who are not so affected. The differences in chest radiograph appearance at 28 days and 36 weeks PMA between those who frequently wheezed and those who did not were greater than the differences in chest radiograph appearance between those who frequently coughed and those who did not. The same method was used to detect cough and wheeze-that is, parents reported their infant's symptoms to a paediatricianthus this is unlikely to have caused the difference in the results. The likely explanation for the difference is that cough is a non-specific symptom, which can be associated with upper as well as lower respiratory tract disorders, whereas wheeze in preterm infants is responsive to bronchodilator treatment ${ }^{3}$ and reflects airway dysfunction. We do not feel our results have been subjected to recall bias by parents of infants who

\begin{tabular}{|c|c|c|}
\hline Predictor & ROC area & $\mathrm{p}$ Value \\
\hline Gestational age & 0.54 & 0.47 \\
\hline Birth weight & 0.49 & 0.81 \\
\hline \multicolumn{3}{|l|}{ Maternal smoking } \\
\hline In pregnancy & 0.52 & 0.59 \\
\hline Affer birth & 0.49 & 0.76 \\
\hline Family history of atopy & 0.43 & 0.12 \\
\hline \multicolumn{3}{|l|}{ Duration of } \\
\hline Ventilation & 0.56 & 0.22 \\
\hline Supplementary oxygen & 0.55 & 0.41 \\
\hline \multicolumn{3}{|l|}{ Oxygen dependency } \\
\hline At 28 days & 0.51 & 0.67 \\
\hline At 36 weeks & 0.54 & 0.28 \\
\hline At discharge & 0.55 & 0.23 \\
\hline \multicolumn{3}{|c|}{ Chest radiograph score at 28 days } \\
\hline Total & 0.55 & 0.31 \\
\hline Fibrosis & 0.56 & 0.19 \\
\hline Cystic elements & 0.52 & 0.44 \\
\hline Hyperinflation & 0.50 & 0.94 \\
\hline \multicolumn{3}{|c|}{ Chest radiograph score at 36 weeks PMA } \\
\hline Total & 0.57 & 0.15 \\
\hline Fibrosis & 0.58 & 0.12 \\
\hline Cystic elements & 0.54 & 0.12 \\
\hline Hyperinflation & 0.51 & 0.77 \\
\hline
\end{tabular}

had required prolonged ventilation, who might be most worried about the development of subsequent respiratory symptoms and hence more likely to recall them. When "days ventilated" and total chest radiograph score were modelled together, however, the relation of the duration of ventilation and frequent wheeze became non-significant, but the relation with the total chest radiograph score remained significant. This shows that the relation between the total chest radiograph score and wheeze was not explained by the number of days ventilated, whereas the association between frequent wheeze and duration of ventilation was explained by the chest radiograph score.

We have previously shown that only a small minority of very preterm infants had no chest radiograph abnormalities at 36 
weeks PMA. ${ }^{7}$ Forty seven of the infants included in that study $^{7}$ had had chest radiographs at 28 days and 36 weeks PMA. Comparison of the paired radiographs showed that the appearance had significantly deteriorated with increasing postnatal age, suggesting continuing morbidity. It is therefore not surprising that we found the chest radiograph appearance at 36 weeks PMA, rather than at 28 days of age, to be significantly related to frequent wheeze at follow up.

Only $20 \%$ of the infants frequently wheezed and $24 \%$ frequently coughed at follow up at 6 months of age. In a previous study, ${ }^{11}$ we had found almost $50 \%$ of preterm infants to be symptomatic at follow up. The two populations, however, differed with respect to their maturity at birth but, as those in the present study were generally born at earlier gestations, a greater proportion would have been predicted to be symptomatic. ${ }^{1}$ The present sample had all been entered into the randomised trial comparing high volume strategy HFO with conventional ventilation for a minimum of five days. The follow up of that trial (UKOS) is continuing, and therefore the trial code has not been broken with respect to the follow up data, but it is expected that about half of the infants in this study would have received HFO. It is possible that use of HFO may have favourably influenced the results, but in the UKOS trial overall no significant difference was seen between the two treatment arms with regard to the occurrence of chronic lung disease. ${ }^{12}$ The difference in the incidence of symptoms between the earlier ${ }^{11}$ and the present study may be explained by use of dissimilar criteria for diagnosis of symptom status. Previously, positive symptom status was diagnosed if the infant wheezed or coughed for at least three days a week for a four week period. In the present study, infants were only described as frequently wheezing or coughing if this occurred at least once a week over six months. Using the latter criterion, we were identifying the most severely affected infants and thus those likely to benefit from treatment.

The infants who frequently wheezed had higher chest radiograph scores for fibrosis/interstitial shadows and cystic elements. Previous studies ${ }^{6}{ }^{13}$ have also reported an association between the presence of fibrosis/interstitial shadows and cystic elements on chest radiographs and respiratory morbidity at follow up. At 28 days of age, infants with the highest chest radiograph scores were noted to be the only ones who had cystic elements and/or interstitial changes present on their chest radiographs. ${ }^{6}$ A high score was associated with oxygen dependency at 28 days and an abnormal airway resistance at 6 months of age. ${ }^{6}$ Infants with interstitial shadows on the chest radiograph have also been shown to be at increased risk of developing hyperinflation and airways obstruction even at 8-10 years of age. ${ }^{13}$ Histopathological staging of changes in the lungs of patients with respiratory distress syndrome who died after receiving mechanical ventilation showed that interstitial change progressing to fibrosis was the most common abnormality and correlated best with radiograph staging. ${ }^{14}$ As the presence of interstitial shadows/fibrosis has been shown to be important with regard to outcome, ${ }^{6}{ }^{13} 14$ we had weighted the scoring system accordingly by recording the presence of such abnormalities in four zones. We now show, by construction of ROC curves and comparison of the areas under those curves, that the presence of interstitial shadows/fibrosis on the chest radiograph at 36 weeks PMA performed best with regard to prediction of frequent wheeze. In addition, the chest radiograph score at 36 weeks PMA appeared to be a better predictor than readily available clinical data.

Routine chest radiographs were to be taken at 28 days and 36 weeks PMA as part of the UKOS protocol. Only a proportion of the clinicians, however, complied, as indicated by our finding that only 185 of the 592 survivors had had both chest radiographs and completed six months of follow up. More of the infants included in this study, compared with the survivors overall, had been oxygen dependent at 36 weeks PMA. These data suggest that the clinicians were more willing to take a routine chest radiograph in an infant who was still oxygen dependent. Therefore we felt it was important to undertake a subanalysis in the infants who were not oxygen dependent, as this analysis would inform clinicians whether a routine chest radiograph in such infants would yield useful information. The results suggest that chest radiograph appearance was a better predictor in non-oxygen dependent infants than in the study population overall, but numbers were too small for us to be able to draw firm conclusions.

The chest radiograph score was a sensitive, but less specific, predictor of frequent wheeze. We therefore conclude that an abnormal chest radiograph appearance at 36 weeks PMA in a very immature infant should alert the clinician to the likelihood of frequent wheeze at follow up. Once such an infant becomes symptomatic, early administration of prophylactic medication should be considered.

\section{ACKNOWLEDGEMENTS}

MT, AJ, and EL were supported by the Medical Research Council. The UKOS trial is funded by the Medical Research Council. We thank Ms Sue Williams for secretarial assistance.

\section{Authors' affiliations}

M Thomas, A Greenough, Department of Child Health, King's College Hospital, London, UK

M A Johnson, S Calvert, Department of Child Health, St George's Hospital Medical School, London, UK

E Limb, J L Peacock, Public Health Sciences, St George's Hospital Medical School

N Marlow, Department of Child Health, University Hospital, Nottingham, UK

\section{REFERENCES}

1 Greenough A, Giffin FJ, Yüksel B. Respiratory morbidity in preschool children born prematurely and its relationship to adverse neonatal events. Acta Paediatr 1996;85:772-7.

2 Yüksel B, Greenough A. Relationship of symptoms to lung function abnormalities in preterm infants at follow-up. Pediatr Pulmonol 1991:11:202-6.

3 Yüksel B, Greenough A. Ipratropium bromide for symptomatic preterm infants. Eur J Pediatr 1991;150:854-7.

4 Yüksel B, Greenough A. Inhaled sodium cromoglycate for preterm children with respiratory symptoms at follow-up. Respir Med 1992:86:131-4.

5 Yüksel B, Greenough A. Randomized trial of inhaled steroids in preterm infants symptomatic at follow-up. Thorax 1992;47:910-13.

6 Yüksel B, Greenough A, Karani J, et al. Chest radiograph scoring system for use in preterm infants. BrJ Radiol 1991:64:1015-18.

7 Greenough A, Dimitriou G, Johnson AH, et al. The chest radiograph appearances of very premature infants at 36 weeks post conceptional age. Br J Radiol 2000;73:366-9

8 Greenough A, Kavvadia K, Johnson AH, et al. A simple chest radiograph score to predict chronic lung disease in prematurely born infants. Br J Radiol 1999;72:530-3.

9 Altman DG, Bland JM. Diagnostic tests 3: receiver operator characteristic plots. BM 1994;309:188.

10 Hanley JA, McNeil BJ. The meaning and use of the area under a receiver operating characteristic (ROC) curve. Radiology 1982;143:29-36.

11 Greenough A, Maconochie I, Yüksel B. Recurrent respiratory symptoms in the first year of life following preterm delivery. J Perinat Med 1990;18:489-94.

12 Johnson AH, Peacock JL, Greenough A, et al. High frequency oscillatory ventilaton for the prevention of chronic lung disease of prematurity. $N$ Engl J Med 2002;347:633-42

13 Mortensson W, Andreasson B, Lindroth M, et al. Potential of early ches roentgenographic examination in ventilator treated newborn infants to predict future lung function and disease. Pediatr Radiol 1989:20:41-4

14 Edwards D, Colby T, Northway W. Radiographic-pathologic correlation in bronchopulmonary dysplasia. J Pediatr 1979;95:834-6. 